\title{
The clinical characteristics of three subgroups of non-functioning pituitary adenomas
}

\author{
Adam Hull ${ }^{1,2}$, Stephen D. Milhollin ${ }^{1}$, John E. Vena ${ }^{1}$, David V. LaBorde ${ }^{3}$ \\ ${ }^{1}$ College of Public Health, University of Georgia, Athens, USA \\ ${ }^{2}$ School of Medicine, Philadelphia College of Osteopathic Medicine-Georgia Campus, Suwanne, USA \\ ${ }^{3}$ School of Medicine, Emory University, Atlanta, USA \\ Email: Adamhu@pcom.edu
}

Received 15 December 2013; revised 15 January 2014; accepted 21 January 2014

Copyright (C) 2014 Adam Hull et al. This is an open access article distributed under the Creative Commons Attribution License, which permits unrestricted use, distribution, and reproduction in any medium, provided the original work is properly cited. In accordance of the Creative Commons Attribution License all Copyrights @ 2014 are reserved for SCIRP and the owner of the intellectual property Adam Hull et al. All Copyright (C) 2014 are guarded by law and by SCIRP as a guardian.

\section{ABSTRACT}

Introduction: Despite the fact that non-functioning pituitary adenomas do not overproduce hormones, many will stain positive for a particular pituitary hormone, which can be used to differentiate these adenomas into subgroups. If these different sub-groups behave differently in terms of post surgical progression of disease (PSPD) rates or other clinical variables, then better treatment and prognosis could be predicted. Methods: This was a retrospective cohort study. Patients who have undergone surgery for removal of a non-functioning pituitary adenoma at Emory University Hospital served as the source for all data used in this study $(n=184)$. Data were collected from a database of electronic medical records (EMRs) for these patients in 2010 documenting clinical and demographic variables including treatment and PSPD. Results: Risk for PSPD did not differ by adenoma subtypes: follicle-stimulating hormone (FSH+), luteinizing hormone $(\mathrm{LH}+)$, or those that do not stain positive for any hormone (non-functioning, or $\mathrm{NF}-)(\mathrm{p}=\mathbf{0 . 9 7 1})$. There were two clinical characteristics statistically related to adenoma subtype: altered mental status and the anterior-posterior (AP) dimension of pre-operative adenomas. PSPD was related to several clinical characteristics, including gender, previous adenoma, post-operative residual, and follow-up time.

\section{KEYWORDS}

Non-Functioning Pituitary Adenomas; Clinical Characteristics; Recurrence

\section{INTRODUCTION}

Non-functioning pituitary adenomas are a subclass of pituitary adenomas which do not directly contribute to hormone overproduction. These types of adenomas account for approximately $30 \%$ of all pituitary adenomas, and are the most common adenomas of the anterior pituitary [1,2]. Although these adenomas do not contribute to hormone overproduction, many non-functioning pituitary adenomas $(40 \%-79 \%)$ will stain positive for a particular pituitary hormone [3]. These pituitary hormones are growth hormone, thyroid-stimulating hormone, adrenocorticotropic hormone, prolactin, luteinizing hormone, follicle-stimulating hormone, melanocyte-stimulating hormone, oxytocin, and antidiuretic hormone. During the course of this analysis, three types of adenomas were examined: those which stain positive for folliclestimulating hormone $(\mathrm{FSH}+)$ or luteinizing hormone $\left(\mathrm{LH}^{+}\right)$, or those that do not stain positive for any hormone (non-functioning, or $\mathrm{NF}^{-}$). These three were chosen because of both their high frequency and genetic differences [2].

A 2005 report in "Cancer Research" reported finding from an analysis of 19 surgical specimens, eleven nonfunctioning pituitary adenoma and eight normal pituitary gland specimens. The paper describes a protocol that utilized GeneChip microarray analysis to elucidate three distinct non-functioning pituitary adenoma gene and protein expression patterns (described as "molecular fingerprints”): i) immunohistochemistry (IHC) positive for luteinizing hormone $(\mathrm{LH}+)$ only; ii) non-functioning adenomas with IHC positive for follicle stimulating hormone $(\mathrm{FSH}+)$ only; and iii) non-functioning adenomas with negative IHC (NF-). Microarray results were validated with the reverse transcription real-time quantitative PCR, using a larger number of non-functioning adenomas. Proteomic analysis was also used to examine the protein expression of these nonfunctioning adenomas. 
The findings provided new insight into the pathogenesis and molecular classification of nonfunctioning pituitary adenomas [2].

Although non-functioning pituitary adenomas are the most common type of pituitary adenoma, they are not always recognized as such, due to a lack of symptoms from a large portion of these adenomas. According to a meta-analysis performed by Molitch et al., the prevalence of non-functioning adenoma is approximately $11 \%$ of all adenomas; with the highest individual study showing a prevalence of $27 \%$ [4]. These adenomas can cause a variety of neurological symptoms, simply due to mass effect. These symptoms commonly include visual field defects, pituitary insufficiency, and severe headaches. Although a patient may not have symptoms when a nonfunctioning pituitary adenoma is detected, the chances of symptoms become greater if the adenoma continues to grow locally.

There are a number of different types of treatments used to treat non-functioning pituitary adenomas. The most common treatment, especially for adenomas larger in size, is surgery. Surgery is the only treatment strategy that has consistently been shown to improve health and alleviate symptoms. However, in $30 \%-50 \%$ of the nonfunctioning pituitary adenomas that are treated, adenoma residue is left that may regrow and cause further problems [5-9]. The goal of surgery is to remove as much adenoma as safely possible, and to protect healthy pituitary tissue surrounding the adenoma [10]. The size and location of the adenoma, as well as the experience of the surgeon performing the operation play a large role in the success of surgical treatment. Radiotherapy is a second and/or additional modality for the treatment of adenomas. Radiotherapy is commonly used to treat smaller adenomas where surgery may not be indicated. Postoperative radiotherapy is also used to reduce the amount of residual and to prevent and/or reduce regrowth. However, utilizing this form of treatment exposes patients to all the risks associated with radiotherapy [11]. Deferring radiotherapy for patients with complete or near-complete resection seems to be a safe and prudent approach. Recurrences may be detected early with high-resolution imaging, and treated effectively with radiation at the time of recurrence [12]. Somatostatin analogues (SSA) and dopamine agonists (DA) are other types of treatment which can be used for non-functioning pituitary adenomas. Over the years, many different SSAs and DAs have been used with varying results. Overall, these treatments are found to be more proficient at preventing adenoma growth rather than reducing the adenoma size, and are therefore, a less practical treatment for adenomas which are already causing symptoms through mass effects [13]. The last option is always abstaining from treatment and allowing the natural course of the non-functioning pitui- tary adenomas to proceed. One study showed that 50\% of patients experienced adenoma growth at 85 months on average. Of those who experienced an increase in adenoma size, 6 out of 28 reported visual field defects and required surgery [3]. Though there are many different options for treating this disease, surgery is generally the option of choice since it is the only one shown to consistently reduce mass effects.

In patients who are not operated on or otherwise treated for pituitary macroadenomas, the quality of life has been shown to decrease. Therefore, some type of treatment is almost always needed [14]. However, patients that receive surgical treatment for non-functioning pituitary macroadenomas have also been shown to have a significant decrease in quality of life [15]. The effects of surgery on overall quality of life are unclear, and may differ greatly from patient to patient. Additionally, surgery itself may also worsen, rather than improve the quality of life in patients treated for non-functioning pituitary adenomas $[16,17]$. Although the effects of treatment on quality of life are not always obvious, each patient must be evaluated individually to assess the effects of this association. It is only when treatment will positively affect quality of life that it should be employed. Thus, surgical treatment should only be employed in scenarios that preserve patients' quality of life.

\section{OBJECTIVE}

The objective of this analysis was to examine the clinical features reported for patient undergoing surgical intervention for non-functioning pituitary adenomas at a single tertiary academic medical center in the southeastern US that underwent IHC analysis of their surgical specimens in order to determine whether certain clinical characteristics are associated with a particular IHC subtype $\left(\mathrm{LH}+, \mathrm{FSH}^{+}\right.$, or $\left.\mathrm{NF}-\right)$ of non-functioning pituitary adenomas.

\section{MATERIALS AND METHODS}

\subsection{Subject Population}

The study population consisted of 184 patients that underwent surgical intervention between 1984 and 2009 for a primary or recurrent non-functioning pituitary adenoma at Emory University Hospital, a tertiary academic medical center in the southeastern US. Inclusion criteria required that the adenomas: i) were classified as non-functioning, and ii) had a verified immunohistochemistry (IHC) showing the expression of $\mathrm{LH}^{+}, \mathrm{FSH}^{+}$, or $\mathrm{NF}^{-}$, those subgroups shown to have differing molecular fingerprints. All patients must have: i) undergone surgical resection and ii) had a follow up period of one or more days post-operatively. 


\subsection{Study Design}

This was a retrospective cohort analysis. Institutional Review Board (IRB) approval was obtained for the study. None of the investigators reported any conflicts of interests. The research was not funded by any individual nor any agency in the public, commercial, or non-profit sectors.

184 patients treated over the past 11 year study period that met inclusion criteria were examined. Non-functioning pituitary adenoma classification and non-functioning pituitary adenoma IHC subtypes (NFPAISs) were verified per routine post-surgical IHC. Alpha subunit analysis was not performed. Based on the verified IHC results, the cohort was stratified into three NFPAISs: $\mathrm{LH}+, \mathrm{FSH}+$ and $\mathrm{NF}^{-}$. Any adenoma that stained positive for a hormone other than LH or FSH was excluded from the study consistent with the protocol used by Moreno et $a l$. These included adenomas with positive IHC stains for growth hormone, adrenocorticotropic hormone, thyroid-stimulating hormone, prolactin, or any combination of the above. A chart review was then conducted on this sample population. Data on a number of clinical characteristics and other patient variables, listed in Table 1, were collected. Data was input into a Microsoft Excel file.

\subsection{Statistical Analysis}

Once data collection was complete, the data set was imported into STATA 12 software for statistical analysis. Comprehensive information on all clinical variables was unattainable for the entire population. Patients with incomplete data were only included in statistical analysis if they had a recorded entry for the variable being analyzed. No use of randomization or experimental intervention was performed during the study. An alpha level $<0.05$ was considered significant.

The data was analyzed for outliers using graphical residual plots, and one patient was removed from the analysis due to surgery date falling outside of study parameters. Frequencies and measures of association were determined. A Pearson's chi-squared test was used to determine an association between immunohistochemical subtype (Table 1), adenoma post-surgical progression of disease (PSPD) (Table 2), and all covariates at $\alpha=0.05$. Logistic regression was used to predict adenoma PSPD from all covariates. Each variable was analyzed for a sufficient number of PSPD and a minimal amount of missing data for each variable.

\section{RESULTS}

Table 1 is a summary of the study population stratified by NFPAIS. A 183 total cases of pituitary adenomas were examined in this study. The most common type of adenoma was NF- with 127 cases, next was FSH+ with 31 cases, and finally LH+ 25 cases. The average age of the population at time of operation was 56 years. Age ranged from 20 to 84 years-old at the time of operation. There were 105 male cases (57.38\%) and 78 females cases (42.62\%). Of the patients who listed their race, 71 participants (50.00\%) identified as white, 67 (47.18\%) identified as black, and 4 (2.82\%) identified as other. Of the 4 pre-existing medical conditions assessed, the most common was hypertension at $47.78 \%(\mathrm{~N}=86)$. Cancer was next at $20.22 \%(\mathrm{~N}=36)$, followed by diabetes with $19.44 \%(\mathrm{~N}=35)$, and then previous adenoma at $7.30 \%$ $(\mathrm{N}=13)$. The most common presenting symptom was visual deficits with 126 cases (72.41\%), followed by headaches with 79 cases (45.14\%). Based on pre-operative magnetic resonance imaging pituitary protocol (MRI-PP) study radiology dictations, the average adenoma volume, including all types was $8.81 \mathrm{~cm}^{3}$, with the smallest adenoma being $0.51 \mathrm{~cm}^{3}$ and the largest adenoma measuring $61.37 \mathrm{~cm}^{3}$. Residual adenoma, based on a review of the immediate post-operative pituitary protocol MRIs done on study participants, was present in 77 cases (45.83\%). During post-operative follow up, there were 15 cases of PSPD (8.93\%) as determined via comparison of the immediate post-operative imaging with subsequent imaging during the follow up period. The average follow-up time in days was 977 and the range varied from 1 day to 7985 days.

Table 1 presents the statistical significance of each variable in relation to the three types of adenomas. NFPAIS was not found to have a significant association with PSPD. There were 15 instances of PSPD; 11 instances in NF- type adenomas, 2 in LH, and 2 in FSH (p $=0.971$ ). Neither sex nor race was found to be significant with p-values of 0.45 and 0.476 , respectively. No prior medical conditions were found to be significant. Altered mental status, was statistically related to NFPAIS. There were 7 instances of altered mental status in NFtype adenomas (5.93\%), 7 in $\mathrm{LH}+(22.58 \%)$, and 2 in FSH+ (8.70\%); $(p=0.018)$. Associations between NFPAIS and visual defect, headache, and decreased/loss of libido were not found to be significant ( $p=0.955$, $p=$ $0.913, p=0.699$, respectively). When adenoma size was examined anterior-posterior length was found to be different between NFPAISs at a level of statistical significance ( $\mathrm{p}<0.001)$.

Table 2 shows us an analysis of the PSPD variable in relation to all other variables gathered in this study. When examining the demographics, age and sex were associated with PSPD at a level of significance ( $p=0.020$ and 0.014 , respectively), but race was not ( $\mathrm{p}=0.585$ ). There were 4 instances of PSPD in males $(26.67 \%$ of all instances of PSPD) and 11 in females (73.33\%). The only pre-existing medical condition which was sta- 
Table 1. Baseline clinical characteristics in 183 patients by NFPAIS.

\begin{tabular}{|c|c|c|c|c|c|c|c|c|c|}
\hline \multicolumn{10}{|c|}{ Non-functioning pituitary adenoma IHC subtypes } \\
\hline \multirow{2}{*}{$\begin{array}{c}\text { Patient Information } \\
\text { Demographics }\end{array}$} & \multicolumn{2}{|c|}{ NF- } & \multicolumn{2}{|c|}{ FSH+ } & \multicolumn{2}{|c|}{$\mathbf{L H}+$} & \multicolumn{2}{|c|}{ Total } & \multirow[t]{2}{*}{ P-Value } \\
\hline & $\mathbf{N}$ & $\%$ & $\mathbf{N}$ & $\%$ & $\mathbf{N}$ & $\%$ & $\mathbf{N}$ & $\%$ & \\
\hline Age $(\mathrm{N}=183)$ & & & & & & & & & 0.697 \\
\hline$($ mean $=56)<40$ & 19 & 14.96 & 5 & 16.13 & 3 & 12.00 & 27 & 14.75 & \\
\hline $40-49$ & 20 & 15.75 & 6 & 19.35 & 5 & 20.00 & 31 & 16.94 & \\
\hline $50-59$ & 30 & 23.62 & 5 & 16.13 & 9 & 36.00 & 44 & 24.04 & \\
\hline$>60$ & 58 & 45.67 & 15 & 48.39 & 8 & 32.00 & 81 & 44.26 & \\
\hline Gender ( $\mathrm{N}=183)$ & & & & & & & & & 0.450 \\
\hline Female & 55 & 43.31 & 15 & 48.39 & 8 & 32.00 & 78 & 42.62 & \\
\hline Race $(\mathrm{N}=142)$ & & & & & & & & & 0.476 \\
\hline White & 52 & 52.00 & 7 & 35.00 & 12 & 54.55 & 71 & 50.00 & \\
\hline Black & 45 & 45.00 & 13 & 65.00 & 9 & 40.91 & 67 & 47.18 & \\
\hline Other & 3 & 3.00 & 0 & 0.00 & 1 & 4.55 & 4 & 2.82 & \\
\hline \multicolumn{10}{|l|}{ Pre-existing Condition } \\
\hline Diabetes $(\mathrm{N}=180)$ & 25 & 20.16 & 6 & 19.35 & 4 & 16.00 & 35 & 19.44 & 0.891 \\
\hline Cancer $(\mathrm{N}=178)$ & 27 & 22.13 & 6 & 19.35 & 3 & 12.00 & 36 & 20.22 & 0.512 \\
\hline Previous Adenoma $(\mathrm{N}=178)$ & 7 & 5.74 & 4 & 12.90 & 2 & 8.00 & 13 & 7.30 & 0.388 \\
\hline \multicolumn{10}{|l|}{ Pre-Op MRI } \\
\hline Adenoma volume $\left(\mathrm{cm}^{3}\right)$ & & & & & & & & & 0.067 \\
\hline$(\mathrm{N}=127 ;$ mean $=8.81)<3.5$ & 31 & 38.27 & 4 & 15.38 & 4 & 20.00 & 39 & 30.71 & \\
\hline $3.5-7.0$ & 22 & 27.16 & 7 & 26.92 & 3 & 15.00 & 32 & 25.20 & \\
\hline $7.1-10.5$ & 12 & 14.81 & 4 & 15.38 & 6 & 30.00 & 22 & 17.32 & \\
\hline$>10.5$ & 16 & 19.75 & 11 & 42.31 & 7 & 35.00 & 34 & 26.77 & \\
\hline \multicolumn{10}{|l|}{ Post-Op MRI } \\
\hline Residual (N = 168) & 55 & 45.83 & 11 & 42.31 & 11 & 50.00 & 77 & 45.83 & 0.868 \\
\hline $\operatorname{PSPD}(\mathrm{N}=168)$ & 11 & 9.17 & 2 & 7.69 & 2 & 9.09 & 15 & 8.93 & 0.971 \\
\hline \multicolumn{10}{|l|}{ Follow-up time } \\
\hline Days of follow-up $(\mathrm{N}=183)$ & & & & & & & & & 0.366 \\
\hline$($ mean $=977)<365$ & 41 & 32.28 & 12 & 38.71 & 8 & 32.00 & 61 & 33.33 & \\
\hline $365-1095$ & 29 & 22.83 & 8 & 25.81 & 9 & 36.00 & 46 & 25.14 & \\
\hline $1096-1826$ & 27 & 21.26 & 8 & 25.81 & 2 & 8.00 & 37 & 20.22 & \\
\hline$>1826$ & 30 & 23.62 & 3 & 9.68 & 6 & 24.00 & 39 & 21.31 & \\
\hline \multicolumn{10}{|l|}{ Presenting symptoms } \\
\hline Headache $(\mathrm{N}=175)$ & 53 & 44.17 & 15 & 48.39 & 11 & 45.83 & 79 & 45.14 & 0.913 \\
\hline
\end{tabular}


Continued

\begin{tabular}{|c|c|c|c|c|c|c|c|c|c|}
\hline Visual Defects $(\mathrm{N}=174)$ & 87 & 73.11 & 22 & 70.97 & 17 & 70.83 & 126 & 72.41 & 0.955 \\
\hline Altered mental status $(\mathrm{N}=172)$ & 7 & 5.93 & 7 & 22.58 & 2 & 8.70 & 16 & 9.30 & 0.018 \\
\hline Decreased/loss of libido $(\mathrm{N}=172)$ & 19 & 16.10 & 7 & 22.58 & 4 & 17.39 & 30 & 17.44 & 0.699 \\
\hline \multicolumn{10}{|l|}{ Adenoma dimensions (cm) } \\
\hline Anterior-posterior & & & & & & & & & $<0.001$ \\
\hline$(\mathrm{N}=128 ;$ mean $=2.3)<2.0$ & 38 & 46.34 & 2 & 7.69 & 4 & 20.00 & 44 & 34.38 & \\
\hline $2.0-2.5$ & 28 & 34.15 & 12 & 46.15 & 6 & 30 & 46 & 35.94 & \\
\hline $2.6-3.0$ & 7 & 8.54 & 10 & 38.46 & 8 & 40.00 & 25 & 19.53 & \\
\hline$>3.0$ & 9 & 10.98 & 2 & 7.69 & 2 & 10.00 & 13 & 10.16 & \\
\hline Transverse & & & & & & & & & 0.492 \\
\hline$(\mathrm{N}=127 ;$ mean $=2.32)<2.0$ & 30 & 37.04 & 7 & 26.92 & 3 & 15.00 & 40 & 31.50 & \\
\hline $2.0-2.5$ & 30 & 37.04 & 11 & 42.31 & 9 & 45.00 & 50 & 39.39 & \\
\hline $2.6-3.0$ & 11 & 13.58 & 5 & 19.23 & 3 & 15.00 & 19 & 14.96 & \\
\hline$>3.0$ & 10 & 12.35 & 3 & 11.54 & 5 & 25.00 & 18 & 14.17 & \\
\hline Craniocaudad & & & & & & & & & 0.361 \\
\hline$(\mathrm{N}=148 ;$ mean $=2.53)<2.0$ & 30 & 31.25 & 5 & 16.67 & 5 & 22.73 & 40 & 27.03 & \\
\hline $2.0-2.5$ & 34 & 35.42 & 9 & 30.00 & 7 & 31.82 & 50 & 33.78 & \\
\hline $2.6-3.0$ & 18 & 18.75 & 6 & 20.00 & 5 & 22.73 & 29 & 19.59 & \\
\hline$>3.0$ & 14 & 14.58 & 10 & 33.33 & 5 & 22.73 & 29 & 19.59 & \\
\hline TOTAL & 127 & 69.40 & 31 & 16.94 & 25 & 13.66 & 183 & 100.00 & \\
\hline
\end{tabular}

tistically significant with PSPD was a history of previously treated pituitary adenoma $(p=0.005)$. In the 13 total cases which had a previously treated adenoma, there were 4 instances of PSPD following the surgical procedure qualifying the subject for inclusion in this study (26.67\%). Pre-op adenoma volume did not have a significant association with PSPD ( $\mathrm{p}=0.943)$. However, it should be noted that there were no instances of PSPD in adenomas less than $2.61 \mathrm{~cm}^{3}$. Residual adenomas seen on post-operative MRI-PP was significantly related to PSPD ( $p=0.001$ ). In the 15 cases of PSPD, only 2 cases did not show residual adenomas on the post-op MRI-PP. Follow-up time was also related to PSPD ( $p=0.023)$. Those subjects with the longest follow up (>1826 days) had the highest incidence of PSPD $(\mathrm{N}=8)$. Although no presenting symptoms were statistically significant. There were no significant relationships between individual adenoma dimensions and PSPD.

Table 3 presents the logistic regression analyses of all variables in relation to adenoma PSPD. The dependent variable $(\beta 0)$ measures adenoma PSPD, and equals 1 for PSPD and 0 for no PSPD. Since this is a discrete dependent variable, an ordinary least squares regression could be used to fit a linear probability model. However, since this model is heteroskedastic and capable of predicting values beyond $\beta 0=0$ or 1 , we used a logistic regression model to better predict categorical or continuous variables that are potentially associated with adenoma PSPD. After performing a univariate regression analysis for PSPD on all covariates, we found that "age at operation", "sex", "previously treated adenoma", "residual", and "days of follow-up" were all significant, single predictors for PSPD. Although these variables are clearly associated with PSPD, the small number of events among each categorical variable caused an inflation in the standard error and confidence intervals, which limited the interpretability of these findings. After adjusting for and removing insignificant terms, an empirical stepwise selection of our multivariate model ended with two significant predictors and an overall significant model $(\mathrm{p}=0.001)$. The $\beta 1$ coefficient of the final model indicates that the odds of adenoma PSPD increases by 0.001 for every day of follow-up ( $=0.023$; CI $1.00006-1.00008)$. This OR is very close to the null value of 1 , and when rounded, could be interpreted as no effect. Additionally, the $\beta 2$ coefficient indicates that the older patients are at time of operation, the less the odds are for adenoma PSPD ( $p=0.006$; CI 0.909 - 0.984). 
Table 2. Baseline clinical characteristics in 183 patients who underwent resection of NFPAs by PSPD.

\begin{tabular}{|c|c|c|c|c|c|c|c|}
\hline \multicolumn{8}{|c|}{ Post-surgical progression of non-functioning pituitary adenoma } \\
\hline \multirow{2}{*}{$\begin{array}{c}\text { Patient Information } \\
\text { Demographics }\end{array}$} & \multicolumn{2}{|c|}{ No } & \multicolumn{2}{|c|}{ Yes } & \multicolumn{2}{|c|}{ Total } & \multirow[t]{2}{*}{ P-Value } \\
\hline & $\mathbf{N}$ & $\%$ & $\mathbf{N}$ & $\%$ & $\mathbf{N}$ & $\%$ & \\
\hline Age $(\mathrm{N}=168)$ & & & & & & & 0.020 \\
\hline$<40$ & 18 & 11.76 & 6 & 40.00 & 24 & 14.29 & \\
\hline $40-49$ & 27 & 17.65 & 2 & 13.33 & 29 & 17.26 & \\
\hline $50-59$ & 38 & 24.84 & 4 & 26.67 & 42 & 25.00 & \\
\hline$>60$ & 70 & 45.75 & 3 & 20.00 & 73 & 43.45 & \\
\hline Gender ( $N=168)$ & & & & & & & 0.014 \\
\hline Male & 91 & 59.48 & 4 & 26.67 & 95 & 56.55 & \\
\hline Female & 62 & 40.52 & 11 & 73.33 & 73 & 43.45 & \\
\hline Race (N = 132) & & & & & & & 0.585 \\
\hline White & 61 & 51.69 & 6 & 42.86 & 67 & 50.76 & \\
\hline Black & 53 & 44.92 & 8 & 57.14 & 61 & 46.21 & \\
\hline Other & 4 & 3.39 & 0 & 0.00 & 4 & 3.03 & \\
\hline \multicolumn{8}{|l|}{ Pre-existing Condition } \\
\hline Diabetes $(\mathrm{N}=165)$ & 28 & 18.67 & 3 & 20.00 & 31 & 18.79 & 0.900 \\
\hline Cancer ( $\mathrm{N}=163)$ & 29 & 19.59 & 4 & 26.67 & 33 & 20.25 & 0.516 \\
\hline Previous Adenoma $(\mathrm{N}=163)$ & 9 & 6.08 & 4 & 26.67 & 13 & 7.98 & 0.005 \\
\hline Hypertension ( $\mathrm{N}=165)$ & 74 & 49.33 & 7 & 46.67 & 81 & 49.09 & 0.844 \\
\hline \multicolumn{8}{|l|}{ Pre-Op MRI } \\
\hline Adenoma volume $\left(\mathrm{cm}^{3}\right)$ & & & & & & & 0.943 \\
\hline$(\mathrm{N}=114)<3.5$ & 30 & 28.85 & 2 & 20.00 & 32 & 28.07 & \\
\hline $3.5-7.0$ & 26 & 25.00 & 3 & 30.00 & 29 & 25.44 & \\
\hline $7.1-10.5$ & 18 & 17.31 & 2 & 20.00 & 20 & 17.54 & \\
\hline$>10.5$ & 30 & 28.85 & 3 & 30.00 & 33 & 28.95 & \\
\hline \multicolumn{8}{|l|}{ Post-Op MRI } \\
\hline Residual (N = 168) & 64 & 41.83 & 13 & 86.67 & 77 & 45.82 & 0.001 \\
\hline \multicolumn{8}{|l|}{ Follow-up time } \\
\hline Days of follow-up ( $\mathrm{N}=168$ ) & & & & & & & 0.023 \\
\hline$<365$ & 46 & 30.07 & 2 & 13.33 & 48 & 28.57 & \\
\hline $365-1095$ & 40 & 26.14 & 4 & 26.67 & 44 & 26.19 & \\
\hline $1096-1826$ & 36 & 23.53 & 1 & 6.67 & 37 & 22.02 & \\
\hline$>1826$ & 31 & 20.26 & 8 & 53.33 & 39 & 23.21 & \\
\hline \multicolumn{8}{|l|}{ Presenting symptoms } \\
\hline Headache $(\mathrm{N}=160)$ & 62 & 42.47 & 9 & 64.29 & 71 & 44.38 & 0.116 \\
\hline Visual Defects $(\mathrm{N}=159)$ & 110 & 75.86 & 8 & 57.14 & 118 & 74.21 & 0.126 \\
\hline
\end{tabular}




\section{Continued}

\begin{tabular}{|c|c|c|c|c|c|c|c|}
\hline Altered mental status $(\mathrm{N}=157)$ & 10 & 6.99 & 3 & 21.43 & 13 & 8.28 & 0.061 \\
\hline Decreased/loss of libido $(\mathrm{N}=157)$ & 29 & 20.28 & 0 & 0.00 & 29 & 18.47 & 0.062 \\
\hline \multicolumn{8}{|l|}{ Adenoma dimensions (cm) } \\
\hline Anterior-posterior $(\mathrm{N}=115)$ & & & & & & & 0.803 \\
\hline$<2.0$ & 35 & 33.33 & 2 & 20.00 & 37 & 32.17 & \\
\hline $2.0-2.5$ & 38 & 36.19 & 5 & 50.00 & 43 & 37.39 & \\
\hline $2.6-3.0$ & 20 & 19.05 & 2 & 20.00 & 22 & 19.13 & \\
\hline$>3.0$ & 12 & 11.43 & 1 & 10.00 & 13 & 11.30 & \\
\hline Transverse $(\mathrm{N}=114)$ & & & & & & & 0.482 \\
\hline$<2.0$ & 30 & 28.85 & 3 & 30.00 & 33 & 28.95 & \\
\hline $2.0-2.5$ & 42 & 40.38 & 2 & 20.00 & 44 & 38.60 & \\
\hline $2.6-3.0$ & 17 & 16.35 & 2 & 20.00 & 44 & 38.60 & \\
\hline$>3.0$ & 15 & 14.42 & 3 & 30.00 & 18 & 15.79 & \\
\hline Craniocaudad $(\mathrm{N}=133)$ & & & & & & & 0.682 \\
\hline$<2.0$ & 33 & 27.27 & 2 & 16.67 & 35 & 26.32 & \\
\hline $2.0-2.5$ & 39 & 32.23 & 4 & 33.33 & 43 & 32.33 & \\
\hline $2.6-3.0$ & 24 & 19.83 & 4 & 33.33 & 28 & 21.05 & \\
\hline$>3.0$ & 25 & 20.66 & 2 & 16.67 & 27 & 20.30 & \\
\hline TOTAL & 153 & 91.07 & 15 & 8.93 & 168 & 100.00 & \\
\hline
\end{tabular}

Table 3. Logistic regression results for significant covariates $(\alpha<0.05)$.

\begin{tabular}{|c|c|c|c|c|c|c|c|c|c|c|c|}
\hline \multicolumn{12}{|c|}{ Univariate logistic regression predicting post-surgical progression of disease } \\
\hline Adenoma PSPD & $\begin{array}{l}\text { Odds } \\
\text { Ratio }\end{array}$ & $\begin{array}{c}\text { Standard } \\
\text { Error }\end{array}$ & $\mathbf{z}$ & $\mathbf{P}>|\mathbf{z}|$ & $\begin{array}{r}95 \% \text { Co } \\
\text { Inte }\end{array}$ & $\begin{array}{l}\text { fidence } \\
\text { val }\end{array}$ & $\begin{array}{c}\text { Log } \\
\text { Likelihood }\end{array}$ & $\begin{array}{l}\text { Obs } \\
(\mathrm{N})\end{array}$ & LR chi ${ }^{2}$ & $\mathbf{P}>\mathbf{c h i}^{2}$ & Pseudo $\mathbf{R}^{2}$ \\
\hline \multicolumn{12}{|l|}{ No $=$ Base Outcome } \\
\hline \multicolumn{12}{|l|}{ Yes } \\
\hline Sex (Female) & 4.036 & 2.449 & 2.300 & 0.021 & 1.229 & 13.255 & -47.529 & 168 & 6.040 & 0.014 & 0.060 \\
\hline Age at operation & 0.946 & 0.019 & -2.740 & 0.006 & 0.909 & 0.984 & -46.474 & 168 & 8.150 & 0.004 & 0.806 \\
\hline Previous adenoma & 5.616 & 3.806 & 2.550 & 0.011 & 1.488 & 21.196 & -47.351 & 163 & 5.440 & 0.020 & 0.054 \\
\hline Residual post-op tissue & 9.039 & 7.024 & 2.830 & 0.005 & 1.971 & 41.451 & -44.573 & 168 & 11.950 & 0.001 & 0.118 \\
\hline \multicolumn{12}{|c|}{ Logistic regression model predicting post-surgical progression of disease } \\
\hline Adenoma PSPD & $\begin{array}{l}\text { Odds } \\
\text { Ratio }\end{array}$ & $\begin{array}{l}\text { Standard } \\
\text { Error }\end{array}$ & $\mathbf{z}$ & $\mathbf{P}>|\mathbf{z}|$ & $\begin{array}{r}95 \% \text { Co } \\
\text { Inte }\end{array}$ & $\begin{array}{l}\text { fidence } \\
\text { val }\end{array}$ & $\begin{array}{c}\text { Log } \\
\text { Likelihood }\end{array}$ & $\begin{array}{l}\text { Obs } \\
\text { (N) }\end{array}$ & LR chi ${ }^{2}$ & $\mathbf{P}>\mathbf{c h i}^{2}$ & Pseudo $\mathbf{R}^{2}$ \\
\hline Days of follow-up $(\beta 1)$ & 1.001 & 0.001 & 2.280 & 0.023 & 1.00006 & 1.00008 & -43.728 & 168 & 13.640 & 0.001 & 0.135 \\
\hline Age at operation ( $\beta 2)$ & 0.951 & 0.019 & -2.740 & 0.006 & 0.909 & 0.984 & -43.728 & 168 & 13.640 & 0.001 & 0.135 \\
\hline
\end{tabular}




\section{DISCUSSION}

The primary goal of this project was to review three previously described IHC subtypes of pituitary adenomas and investigate if there are any trends which may provide insight into the treatment, natural history, and/or prognosis of these pituitary tumors (Table 1). The main association of interest was whether or not NFPAIS was significantly related to PSPD of the adenoma. No statistical relationship was found $(\mathrm{p}=0.971)$. There were 15 instances of PSPD; 11 instances in NF- type adenomas, 2 in LH, and 2 in FSH. This represents a 8.93\% PSPD rate overall, 9.17\% PSPD rate in NF- adenomas, $7.69 \%$ in $\mathrm{LH}+$ type, and $9.09 \%$ in $\mathrm{FSH}+$. There were similar PSPD rates among all type of adenomas. Another important relationship examined was whether NFPAIS was related to adenoma size. The average volume of adenomas at presentation was $8.81 \mathrm{~cm} 3$, however, no association between adenoma size and NFPAIS was found ( $p=0.067)$.

There were two variables which were found to be significantly related to NFPAIS: altered mental status ( $\mathrm{p}=$ 0.018 ), and the anterior-posterior (AP) dimension of the adenoma ( $p<0.001)$. There were 7 instances of altered mental status in NF- adenomas (5.93\%), 7 in $\mathrm{LH}^{+}$ (22.58\%), and 2 in $\mathrm{FSH}+(8.70 \%)$ showing that the incidence of altered mental status in $\mathrm{LH}+$ adenomas was significantly higher than $\mathrm{NF}-$ or $\mathrm{FSH}+$. This association may be a statistical anomaly as the reasoning behind this increased presentation among $\mathrm{LH}^{+}$adenomas is not clearly understood. When excluding all patients with less than one year of follow up $(\mathrm{N}=61)$, altered mental status no longer remains statistically significant (p-value changed from 0.018 to 0.126 ). This is the only variable which significantly changes when patients with less than one year of follow up are excluded. Another significant association was seen in the AP dimension of the adenoma, with the greatest difference in adenomas with an AP size $<2.0 \mathrm{~cm}$. $46.34 \%$ of NF- adenomas are seen at this size, where as only $7.69 \%$ of $\mathrm{FSH}+$ and $20.00 \%$ of $\mathrm{LH}+$ are within this size. FSH+ and $\mathrm{LH}+$ adenomas tended to have a greater distribution in the larger size categories. Although the interpretability of this finding is limited by a small sample size, these data suggest that NF- adenomas do not grow in the AP diameter as much as the other two types.

PSPD was related to several clinical characteristics, including sex $(\mathrm{p}=0.014)$, residual $(\mathrm{p}=0.001)$, previous adenoma $(\mathrm{p}=0.005)$, and follow-up time $(\mathrm{p}=0.023$; see Table 2). There were 4 instances of PSPD in males which accounted for $4.21 \%$ of the male population and 11 in females representing $15.07 \%$ of the female population, with an overall incidence of 15 and PSPD rate of 8.93\%. These data show that females in the study had a much greater incidence of PSPD than males. However, sex was also significantly associated with previous can- cer, previous adenoma, headache, and loss of libido, and an assessment of confounding was limited due to the small number of PSPD. Further research with a larger number of cases is needed to heighten the external validity of this association. Additionally, there was a clear association between residual and PSPD. If there was significant amount of adenoma that remained after the operation, it could serve as a source of the PSPD. Previous adenomas were also significantly associated with PSPD. This relationship can be explained by the same reasoning underlying the association between residual and PSPD. When the previous adenoma was first treated, if the entire adenoma was not resected, then the remainder could serve as a nidus. Moreover, there was a significant association between PSPD and follow up time. Intuitively, this could be explained by the fact that without follow-up, it would be impossible to assess adenoma PSPD. Unfortunately, many individuals did not return for additional care, and were lost to follow-up. If there was PSPD in these individuals, it is unknown. When excluding all patients with less than one year of follow up $(N=61)$, altered mental status became statistically significant with PSPD (p-value changed from 0.061 to 0.042). Although the change in $\mathrm{p}$-value was not large, it was enough to make this association significant. As with altered mental status and NFPAIS, the reasoning behind this association is unknown.

Our regression model (Table 3) shows that for every day of follow-up, the odds of adenoma PSPD increase by a factor of 0.001 when controlling for age at diagnosis. Although this may seem subtle and perhaps clinically irrelevant, the implications of this finding are augmented when considering the longevity of adenoma progression and the brevity of one day. In fact, when substituting follow-up years into the same regression model, we arrived at an odds ratio (OR) of 1.177; for every year of follow-up, the odds of adenoma PSPD increase by a factor of 0.177 when controlling for age at diagnosis $(\mathrm{p}=$ 0.023, CI: 1.0230, 1.353). The standard errors, p-values, and confidence intervals were the same for follow-up days and years, but we decided to use days in our model in order to maximize the precision of our estimates. Furthermore, for every additional year in age at diagnosis, our model shows that the odds of adenoma PSPD were 0.049 less when controlling for days of follow-up. This could be due to different care-seeking practices, better access to screening, or other age-related behaviors that merit further investigation.

There is a widely acknowledged rule of thumb that logistic regression models should be used with a minimum of 10 outcome events per predictor variable. This criteria was impossible to meet given the low counts of PSPD in our dataset $(n=13)$, which made the predicted coefficients vulnerable to small-sample bias and separation. 
Exact logistic regression was attempted, but was computationally intensive and impractical given our two continuous predictors. Alternatively, the Firth method of penalized regression (a general approach to reducing smallsample bias in maximum likelihood estimations) was used to replicate our model, and the calculated coefficients that were nearly identical to those derived from traditional logistic regression. The final model showed conformity with a linear gradient, was free of collinearity and specification error, and depicted a good fit with the data. When considering the analytic criteria of our methods and results that were followed in our protocol, we find that our model provides accurate and valid predictions.

Given the retrospective nature of this study, there were inherent limitations present. Although our data was collected sufficiently and contained a number of important variables, the temporal relationship of these variables was often difficult to assess. For instance, there was limited information regarding the timeframe of prior medical conditions (previous adenoma diagnoses, visual defects, etc.), and also on complete follow-up histories. With this limitation, the possibility for different biases might arise since adenomas progress slowly and patients require periodic screenings to detect PSPD. Additionally, confounding was a potential issue since we were unable to select patients randomly due to the rarity of pituitary adenomas and the small cohort of these patients with IHC results in the academic center's registry of cases. To control for confounding, we analyzed variables within the strata of PSPD among the unexposed, and with the exposure among without PSPD. Another limitation to this study was the lack of data gathered regarding other treatment modalities the patients may have received. Although surgical resection was an inclusion criteria, adjunct treatment options such as radiation therapy were not considered, and could serve as unknown confounders.

The data for this study were gathered exclusively from electronic medical records (EMR). As the use of EMRs continues to expand, these types of cohort studies can be performed more easily and efficiently than was previously possible. The need for proper documentation and structured interoperable data in EMRs are clearly visible to the researchers during this study. Although a total of 183 participants were in the study, many of the participants did not have complete data on all variables examined.

Pituitary adenomas are and will continue to be a common source of morbidity and mortality. These adenomas are often insidious and identifying them early in their course, when more easily treatable, is a challenge for physicians due to the variability of presenting symptoms. Information that can be gathered on presenting symptoms and differences between the natural history of various subtypes may aid in the early diagnosis of these adenomas and thereby decrease both the associated morbidity and mortality.

\section{REFERENCES}

[1] Alexander, J.M., et al. (1990) Clinically nonfunctioning pituitary tumors are monoclonal in origin. The Journal of Clinical Investigation, 86, 336-340. http://dx.doi.org/10.1172/JCI114705

[2] Moreno, C.S., et al. (2005) Novel molecular signaling and classification of human clinically nonfunctional pituitary adenomas identified by gene expression profiling and proteomic analyses. Cancer Research, 65, 1021410222.

http://dx.doi.org/10.1158/0008-5472.CAN-05-0884

[3] Ebersold, M.J., et al., (1986) Long-term results in transsphenoidal removal of nonfunctioning pituitary adenomas. Journal of Neurosurgery, 64, 713-719. http://dx.doi.org/10.3171/jns.1986.64.5.0713

[4] Chun, M., G.B. Masko, and S. Hetelekidis (1988) Radiotherapy in the treatment of pituitary adenomas. International Journal of Radiation Oncology *Biology* Physics, 15, 305-309. http://dx.doi.org/10.1016/S0360-3016(98)90009-8

[5] Grigsby, P.W., et al. (1988) Prognostic factors and results of radiotherapy alone in the management of pituitary adenomas. International Journal of Radiation Oncology *Biology* Physics, 15, 1103-1110. http://dx.doi.org/10.1016/0360-3016(88)90191-5

[6] Comtois, R., et al. (1991) The clinical and endocrine outcome to trans-sphenoidal microsurgery of nonsecreting pituitary adenomas. Cancer, 68, 860-866.

http://dx.doi.org/10.1002/1097-0142(19910815)68:4<860 ::AID-CNCR2820680431>3.0.CO;2-4

[7] Chandler, W.F. and A.L. Barkan (2008) Treatment of pituitary tumors: A surgical perspective. Endocrinology and Metabolism Clinics of North America, 37, 51-66, viii. http://dx.doi.org/10.1016/j.ecl.2007.10.006

[8] Boelaert, K. and N.J. Gittoes (2001) Radiotherapy for non-functioning pituitary adenomas. European Journal of Endocrinology, 144, 569-575.

http://dx.doi.org/10.1530/eje.0.1440569

[9] Park, P., et al. (2004) The role of radiation therapy after surgical resection of nonfunctional pituitary macroadenomas. Neurosurgery, 55, 100-106; discussion 106-107. http://dx.doi.org/10.1227/01.NEU.0000126885.71242.D7

[10] Colao, A., et al. (2008) Medical therapy for clinically non-functioning pituitary adenomas. Endocrine-Related Cancer, 15, 905-915. http://dx.doi.org/10.1677/ERC-08-0181

[11] Johnson, M.D., Woodburn, C.J. and Vance, M.L. (2003) Quality of life in patients with a pituitary adenoma. Pituitary, 6, 81-87. http://dx.doi.org/10.1023/B:PITU.0000004798.27230.ed

[12] Dekkers, O.M., et al. (2006) Quality of life is decreased after treatment for nonfunctioning pituitary macroadeno- 
ma. The Journal of Clinical Endocrinology and Metabolism, 91, 3364-3369.

http://dx.doi.org/10.1210/jc.2006-0003

[13] Dekkers, O.M., et al. (2007) The natural course of nonfunctioning pituitary macroadenomas. European Journal of Endocrinology, 156, 217-224.

http://dx.doi.org/10.1530/eje.1.02334
[14] Dekkers, O.M., et al. (2006) Observation alone after transsphenoidal surgery for nonfunctioning pituitary macroadenoma. The Journal of Clinical Endocrinology and Metabolism, 91, 1796-1801.

http://dx.doi.org/10.1210/jc.2005-2552 